\title{
Increased understanding of the dynamics and transport in ITB plasmas from multi-machine comparisons
}

\author{
P. Gohil ${ }^{1}$, J. Kinsey ${ }^{2}$, V. Parail ${ }^{3}$, X. Litaudon ${ }^{4}$, T. Fukuda ${ }^{5}$, \\ T. Hoang ${ }^{4}$, \\ for the ITPA Group on Transport and ITB Physics: J. Connor ${ }^{6}$, \\ E.J. Doyle 7 , Yu. Esipchuk ${ }^{8}$, T. Fujita ${ }^{5}$, T. Fukuda ${ }^{5}$, P. Gohil ${ }^{1}$, \\ J. Kinsey ${ }^{2}$, S. Lebedev ${ }^{9}$, X. Litaudon ${ }^{4}$, V. Mukhovatov ${ }^{10}$, J. Rice ${ }^{11}$, \\ E. Synakowski ${ }^{12}$, K. Toi ${ }^{13}$, B. Unterberg ${ }^{14}$, V. Vershkov ${ }^{8}$, \\ M. Wakatani ${ }^{15}$, J. Weiland ${ }^{16}$ \\ and for the International ITB Database Working Group: \\ T. Aniel ${ }^{4}$, Yu.F. Baranov ${ }^{3}$, E. Barbato ${ }^{17}$, A. Bécoulet ${ }^{4}$, \\ C. Bourdelle ${ }^{4}$, G. Bracco ${ }^{17}$, R.V. Budny ${ }^{12}$, P. Buratti ${ }^{17}$, E.J. Doyle ${ }^{7}$, \\ L. Ericsson ${ }^{4}$, Yu. Esipchuk ${ }^{8}$, B. Esposito ${ }^{17}$, T. Fujita ${ }^{5}$, T. Fukuda ${ }^{5}$, \\ P. Gohil ${ }^{1}$, C. Greenfield ${ }^{1}$, M. Greenwald ${ }^{11}$, T. Hahm ${ }^{12}$, \\ T. Hellsten ${ }^{3}$, T. Hoang ${ }^{4}$, D. Hogeweij ${ }^{18}$, S. Ide ${ }^{5}$, F. Imbeaux ${ }^{4}$, \\ Y. Kamada ${ }^{5}$, J. Kinsey ${ }^{2}$, N. Kirneva ${ }^{8}$, X. Litaudon ${ }^{4}$, P. Maget ${ }^{4}$, \\ A. Peeters ${ }^{19}$, K. Razumova ${ }^{8}$, F. Ryter ${ }^{19}$, Y. Sakamoto ${ }^{5}$, H. Shirai ${ }^{5}$, \\ G. Sips ${ }^{19}$, T. Suzuki ${ }^{5}$, E. Synakowski ${ }^{12}$ and T. Takizuka ${ }^{5}$
}

${ }^{1}$ General Atomics, PO Box 85608, San Diego, CA 92186-5608, USA

${ }^{2}$ Lehigh University, Bethlehem, PA 18015, USA

${ }^{3}$ EFDA-JET CSU, Culham Science Centre, Abingdon, Oxon, UK

${ }^{4}$ Association Euratom-CEA, CEA de Cadarache, St Paul lez Durance, France

${ }^{5}$ JAERI, Naka Fusion Research Establishment, Naka, Japan

${ }^{6}$ EURATOM/UKAEA Association, Culham Science Centre, Abingdon, Oxon, UK

${ }^{7}$ University of California, Los Angeles, CA 90095, USA

${ }^{8}$ Kurchatov Institute of Atomic Energy, Moscow, Russia

${ }^{9}$ Ioffe Institute, St Petersburg, Russia

${ }^{10}$ ITER JWS, Naka, Japan

${ }^{11}$ Massachusetts Institute of Technology, Cambridge, MA 02139, USA

${ }^{12}$ Plasma Physics Laboratory, Princeton University, Princeton, NJ 08543, USA

${ }^{13}$ National Institute of Fusion Science, Toki City, Japan

${ }^{14}$ Forschungszentrum Jülich, GmbH, EURATOM-Association, Jülich, Germany

${ }^{15}$ Kyoto University, Kyoto, Japan

${ }^{16}$ Chalmers University and EURATOM-VR association, Gothenburg, Sweden

${ }^{17}$ Associazione EURATOM-ENEA sulla Fusione, C.R. Frascati, Frascati, Italy

${ }^{18}$ FOM Insituut voor Plasmafisica, 'Rijnhuizen', Nieuwegein, The Netherlands

${ }^{19}$ Max-Planck-Institut für Plasmaphysik, EURATOM Association, Garching, Germany

E-mail: gohil@fusion.gat.com

Received 14 November 2002, accepted for publication 30 June 2003

Published 1 August 2003

Online at stacks.iop.org/NF/43/708

\begin{abstract}
Our understanding of the physics of internal transport barriers (ITBs) is being advanced by analysis and comparisons of experimental data from many different tokamaks worldwide. An international database consisting of scalar and two-dimensional profile data for ITB plasmas is being developed to determine the requirements for the formation and sustainment of ITBs and to perform tests of theory-based transport models in an effort to improve the predictive capability of the models. Analysis using the database indicates that: (a) the power required to form ITBs decreases with increased negative magnetic shear of the target plasma, and: (b) the $E \times B$ flow shear rate is close to the linear
\end{abstract}


growth rate of the ion temperature gradient (ITG) modes at the time of barrier formation when compared for several fusion devices. Tests of several transport models (JETTO, Weiland model) using the two-dimensional profile data indicate that there is only limited agreement between the model predictions and the experimental results for the range of plasma conditions examined for the different devices (DIII-D, JET, JT-60U). Gyrokinetic stability analysis (using the GKS code) of the ITB discharges from these devices indicates that the ITG/TEM growth rates decrease with increased negative magnetic shear and that the $E \times B$ shear rate is comparable to the linear growth rates at the location of the ITB.

PACS numbers: 52.25.Fi, 52.55.Fa, 52.65.Tt

\section{Introduction}

The International Tokamak Physics Activity (ITPA) group on transport and internal transport barrier (ITB) physics was recently formed to address issues relating to transport in tokamaks with an emphasis on future burning plasma devices. The group's activities include: (a) examining and compiling experimental results on transport from machines worldwide to understand better the physics of ITB formation and sustainment; (b) developing an international database of ITB experimental results to determine the requirements for the formation and sustainment of ITBs; (c) performing comprehensive tests of theory-based models and simulations using the experimental ITB database (ITBDB), which is a critical and necessary step in validating models and providing a predictive capability for future burning plasmas; (d) identifying experiments to address and resolve critical issues in transport and ITB research; (e) facilitating intermachine ITB experiments and comparisons. In all these areas, utilizing results from multi-machine comparisons should increase confidence and provide consistency in our understanding of plasma transport as well as indicate deficiencies in research and data in certain areas. This paper will present details of the results from the group's activities using the international ITB database in conjunction with the ITBDB working group.

It should also be noted that the work described in this paper is expandable, for example, by increasing the number of transport models to be tested, by increasing the range of plasma conditions and scenarios (e.g. $T_{\mathrm{e}}$ barriers, RF heated discharges, etc) to be examined and, by increasing the range of issues (e.g. electron transport, impurity transport, profile control, etc) to be addressed.

\section{International ITBDB}

The formation and development of the international ITBDB is an important step towards determining the underlying physics of ITB formation and sustainment from results obtained from many different tokamaks. Issues that the ITBDB aims to address include identifying key trends for ITB formation and improving the predictive capability for the confinement characteristics of advanced tokamak plasmas with ITBs. In addition to this, the ITBDB can form the basis for tests of theoretical models in order to assess their predictive capability. The ITBDB variables consist of 134 O-D global and local parameters together with two-dimensional profile data (e.g. profiles of ion temperature, electron temperature, electron density, toroidal rotation, safety factor $(q)$, power deposition). The scalar database contains more than 1000 timeslices from many different tokamaks (ASDEX Upgrade, DIII-D, FTU, EFDA-JET, JET, JT-60U, RTP, T10, TFTR, Tore Supra) as well as 4152 profiles in the profile database.

Preliminary analysis of the O-D ITBDB has focused on the power threshold for ITB formation based on global variables such as $n_{\mathrm{e}}, B_{\mathrm{T}}$, and $I_{\mathrm{p}}$ as well as on local quantities such as the magnetic shear at the foot of the barrier [1-3]. A possible scaling law for the power threshold for dominant ion-heating (i.e. for ion ITBs) has been determined using regression analysis [2]. There is a density dependence and a strong dependence on the minor radius (size scaling) whereas the dependence on the toroidal field is weak. A scaling expression for electron ITBs was also evaluated, showing again strong dependence on the minor radius, but with a very weak density dependence. Overall, for similar parameters, the power required to form electron ITBs (in dominantly electron heated discharges) is approximately a factor of 3-4 lower than for ion ITBs (in dominantly ion heated discharges) when examined over a range of devices. The density dependence of the power threshold for the ion ITBs is evident in many devices where ITB formation is only possible at low or moderate target electron densities since target density can influence the power deposition profile (for NBI heating) and the shape and evolution of the $q$-profile. Similarly, increased minor radius (i.e. machine size) requires higher input power to maintain the required power density for ITB formation. The power threshold dependence on the electron density and minor radius for predominantly NBI discharges indicates that the input power per particle is an important parameter for ITB formation and sustainment. Increased input power per particle can lead to increased pressure gradients and toroidal rotation (for cases where momentum torque is applied), which in turn can increase the $E \times B$ shearing rate so providing for enhanced fluctuation suppression. Further, ITB formation is the result of local changes in transport at any location in the plasma interior and as such is very dependent on local parameters and their evolution, and especially on the radial profiles of various quantities such as the power deposition and $q$-profiles. Future work is therefore required to incorporate the effects of local parameters into the predictive capability for determining the power threshold for ITBs and will be based on providing more profile data into the database.

The O-D ITBDB has also been used to examine the influence of plasma confinement and magnetic shear on ITB formation [4]. Figure 1 shows the amount of heating power per particle just prior to the formation of ion ITBs as a function of the inverse of the normalized central ion poloidal gyroradius 


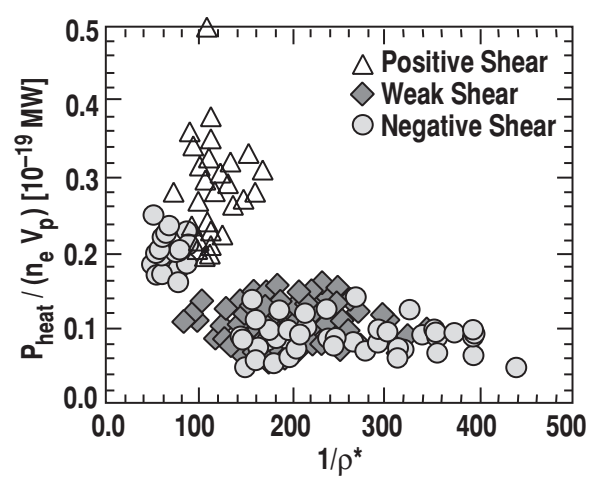

Figure 1. The heating power per particle as a function of $1 / \rho^{*}$ for various $q$-profiles in ASDEX-Upgrade, DIII-D, JET, JT-60U, and TFTR evaluated at the time of ITB formation. The positive shear data only from JT-60U. The negative shear data between $150<1 / \rho^{*}<400$ is also from JT-60U.

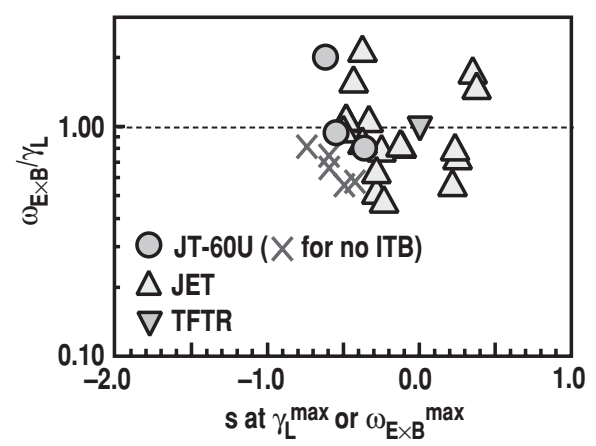

Figure 2. The ratio $\omega_{E \times B} / \gamma_{\mathrm{L}}$ calculated at the time of ITB formation for JT-60U, JET, and TFTR as a function of the local magnetic shear at the maximum of the linear growth rate or $E \times B$ shearing rate (which is close to the location of the ITB).

$\rho_{\theta}^{*}=\rho_{i \theta} / a$ where $a$ is the minor radius. Figure 1 shows that weak or negative magnetic shear is more favourable for ITB formation since, at $1 / \rho^{*}=100-150$, the power required for ITB formation is about 2-3 times lower than for plasmas with positive magnetic shear. This behaviour is consistent with the reduction of the turbulence growth rates by negative magnetic shear which facilitates ITB formation by lowering the input power and $E \times B$ shearing rate required for formation. The $q$-profile has a strong influence on the ITB power threshold and this will be the subject of further discussion in the following sections.

The database was also used to examine the ratio of the $E \times B$ shearing rate, $\omega_{E \times B}$, to the ion temperature gradient (ITG) growth rate, $\gamma_{\mathrm{L}}$, at the time of ITB formation for several devices [5]. Figure 2 indicates that the ratio of $\omega_{E \times B} / \gamma_{\mathrm{L}}$ is close to unity at the time of clear ITB formation in JT-60U and TFTR, whereas results from JET exhibit more scatter, but still satisfy the above inequality within a factor of 2 , given the uncertainties in the calculations of the $E \times B$ shear rates and the growth rates. The $E \times B$ shearing rate was determined from the Hahm-Burrell formulation given in [6] and the ITG growth rates were calculated using the formulation in [7]. The above result is consistent with the paradigm of reduced plasmas transport by $E \times B$ flow shear [8].

\section{Predictive modelling of plasmas with ITBs}

As pointed out in the previous section, the $q$-profile can affect the formation characteristics and requirements of ITBs. In order to obtain a better understanding of the influence of the $q$-profile on ITB formation, discharges from three major tokamaks (DIII-D, JET, and JT-60U) were modelled using various transport models. Pairs of discharges from each device were selected with one discharge of the pair having a weakly reversed shear or monotonic $q$-profile and the other discharge having a strongly reversed central magnetic shear. Modelling of these discharges would then reveal differences due to the $q$-profile and also provide checks of the validity of the theory based transport models. In the preliminary analyses of these discharges, three transport models were considered in this work: the Weiland model [9], the GLF23 model [10], and the JETTO code [11]. In performing the analysis of the above discharges in the ITBDB with the GLF23 model, it was determined that the model contained a serious error when modelling discharges with weak or strongly reversed magnetic shear and moderate to large alpha (normalized pressure gradient) [12]. An error was found in the radial mode damping rate which enters in the mixing length formula for the fluxes in GLF23. It was discovered that the linear growth rates did not compare well with the linear gyrokinetic growth rates from the GKS gyrokinetic code for weak or reversed magnetic shear plasmas contained in the ITB profile database. Therefore, the GLF23 model could not be used in this comparative analysis and efforts were commenced to correct the model. In this respect, performing the multimachine comparative analyses described in this paper allowed the error to be discovered and provided a positive contribution towards improving this model.

The JETTO code is based on an empirical mixed Bohm/gyroBohm transport model [13], which has been previously extensively tested on JET plasmas. This transport model has two main terms contributing to anomalous transport. A Bohm term, which represents the long wavelength part of the turbulence, is suppressed by either a strong $E \times B$ shearing rate or by negative magnetic shear:

$\chi_{\text {Bohm }} \propto\left|\frac{\nabla n_{\mathrm{e}} T_{\mathrm{e}}}{n_{\mathrm{e}} B}\right| \cdot q^{2} \cdot\left|\frac{\nabla T_{\mathrm{e}}}{\mathrm{T}_{\mathrm{e}}}\right|_{a} \cdot H\left(0.05+s-C \cdot \frac{\omega_{E \times B}}{\gamma}\right)$,

where $H(x)$ is a Heaviside step function, $s$ the magnetic shear, $C$ an adjustable numerical factor (of the order of one), $\gamma$ the growth rate, and $\omega_{E \times B}$ is the $E \times B$ shearing rate. A gyroBohm term, which represents short wavelength turbulence (like ETG), is influenced by small magnetic shear:

$$
\chi_{\text {gyroBohm }} \propto \frac{T_{\mathrm{e}}^{3 / 2}}{B^{2}}\left|\frac{\nabla T_{\mathrm{e}}}{T_{\mathrm{e}}}\right| \cdot \frac{|s|}{1+|s|},
$$

rather than by the strong $E \times B$ shearing rate. JETTO uses a simplified version of the growth rate where $\gamma \approx V_{T_{\mathrm{i}}} / q R$ and the $E \times B$ shearing rate is derived from the Hahm-Burrell formulation [6]. All neoclassical quantities (like poloidal rotation, resistivity, and bootstrap current) are calculated by NCLASS [14]. The quoted diffusivities are used for the electron and ion thermal transport only since the actual time dependent experimental profiles of the toroidal rotation and 
electron density were used in the modelling. After the transport reduction, the remaining transport is neoclassical plus gyroBohm.

The latest version of the Weiland ITG/TEM transport model includes magnetic shear effects, off-diagonal elements and $E \times B$ shear stabilization. The Weiland model is an advanced reactive fluid model with the dissipative part of the kinetic resonance omitted (reactive closure) and includes a heuristical implementation of the turbulence suppression by the $E \times B$ shearing rate:

$$
\chi \propto \sum_{k} \frac{\gamma_{k}-\omega_{E \times B}}{k_{\perp}^{2}} \times H\left(\gamma_{k}-\omega_{E \times B}\right),
$$

where $H(x)$ is a Heaviside step function, $\gamma_{k}$ is the characteristic growth rate and $k$ is the characteristic perpendicular wave vector. The model includes parallel ion motion and finite beta effects, but no ETG physics is included. The $\omega_{E \times B}$ rate is determined from the definition of Waltz et al [10] and the growth rate is determined using a single wave vector approximation for $\kappa_{\perp} \rho_{i} \approx 0.3$.

Although JETTO can be run in a fully predictive way (including self-consistent modelling of density, toroidal rotation and safety factor), the actual experimental density profiles and the toroidal rotation profiles were used in the modelling together with the experimental electron and ion temperatures as the initial starting conditions. The actual experimental profiles (for $T_{\mathrm{e}}, T_{\mathrm{i}}, n_{\mathrm{e}}, v_{\phi}$ ) were used as the initial input values in the models after which the model codes were run in a self-consistent, time dependent manner in which the profiles of $T_{\mathrm{e}}$ and $T_{\mathrm{i}}$ were allowed to evolve (whilst using the actual time dependent profiles of $n_{\mathrm{e}}$ and $v_{\phi}$ ) until a quasi-steady state solution for the $T_{\mathrm{e}}$ and $T_{\mathrm{i}}$ profiles is found and these are then the model predictions. The model predictions of the profiles are then compared with the actual experimental time dependent profiles of $T_{\mathrm{e}}$ and $T_{\mathrm{i}}$ (later in time) to determine the level of agreement. Figures 3-5 show the results of predictive modelling of DIII-D, JET, and JT-60U discharges using the JETTO code. The experimental profiles of the ion and electron temperatures are compared to the results from the model for different values of the parameter $C$. For the DIII-D discharge with more strongly reversed central magnetic shear (shot 95989), the predicted $T_{\mathrm{i}}$ profile from the model shows reasonable agreement with the experimental profile (figure $3(d)$ ) whereas the $T_{\mathrm{e}}$ profile indicates clear underestimation of the temperature by the model (figure $3(e)$ ). There is no significant variation of the modelled temperature profiles with the parameter $C$ since the dominant mechanism for the ITB formation in this model is the negative magnetic shear and the relative influence of the $E \times B$ shearing rate is weak for these conditions. For the case of the weak negative shear discharge (shot 87031), the model substantially overestimates both the $T_{\mathrm{i}}$ and $T_{\mathrm{e}}$ profiles compared to the experimental values (figures 3(a) and $(b)$ ). Furthermore, there is a larger variation in the predicted temperature with changes in the parameter $C$. This is a result of the greater influence of the $E \times B$ shearing rate for weak negative shear although the model still incorporates a strong influence from even this lower degree of magnetic shear. For the JET discharge with strong negative central shear (NCS) (shot 53521), there is very good agreement between the model predictions and both the experimental $T_{\mathrm{i}}$ and $T_{\mathrm{e}}$ profiles (figures $4(d)$ and $(e)$ ). The model provides good agreement with both the absolute value of the central temperature and the spatial location of the transport barrier. However, the model substantially overestimates the temperature profiles for the JET discharge with monotonic $q$-profile (shot 46664), particularly the $T_{\mathrm{i}}$ profile (figure 4(a)). This overestimation is very similar to the behaviour for weak negative magnetic shear discharges in DIII-D suggesting that the model is less appropriate for discharges with both weak magnetic shear and strong toroidal rotation or strong pressure gradients. In the case of the JT-60U discharge with monotonic $q$-profile (shot 34487 ), the model clearly fails to reproduce the
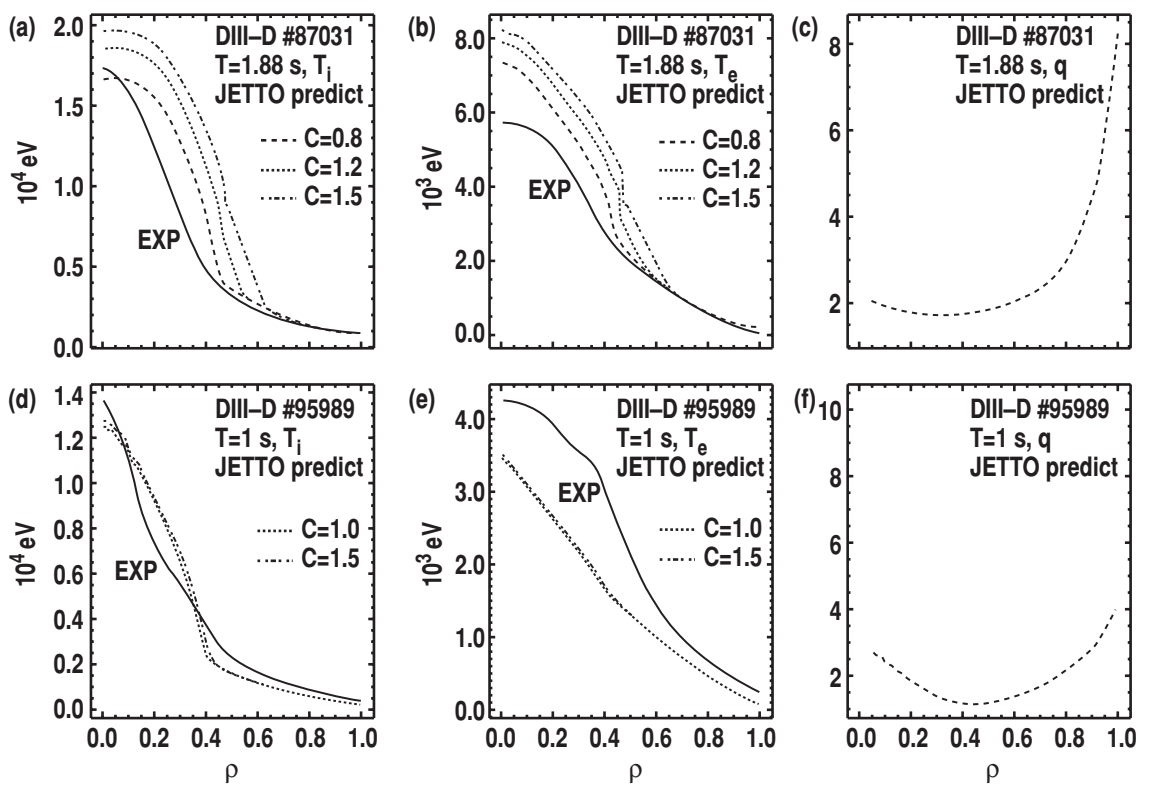

Figure 3. Comparisons between predicted results from the JETTO transport model and experimental results for the $T_{\mathrm{i}}$ and $T_{\mathrm{e}}$ profiles in ITB discharges in DIII-D. The $q$-profiles are the predicted values from JETTO. $(a)-(c)$ correspond to weak negative central magnetic shear whilst $(d)-(f)$ correspond to strong negative magnetic shear. 

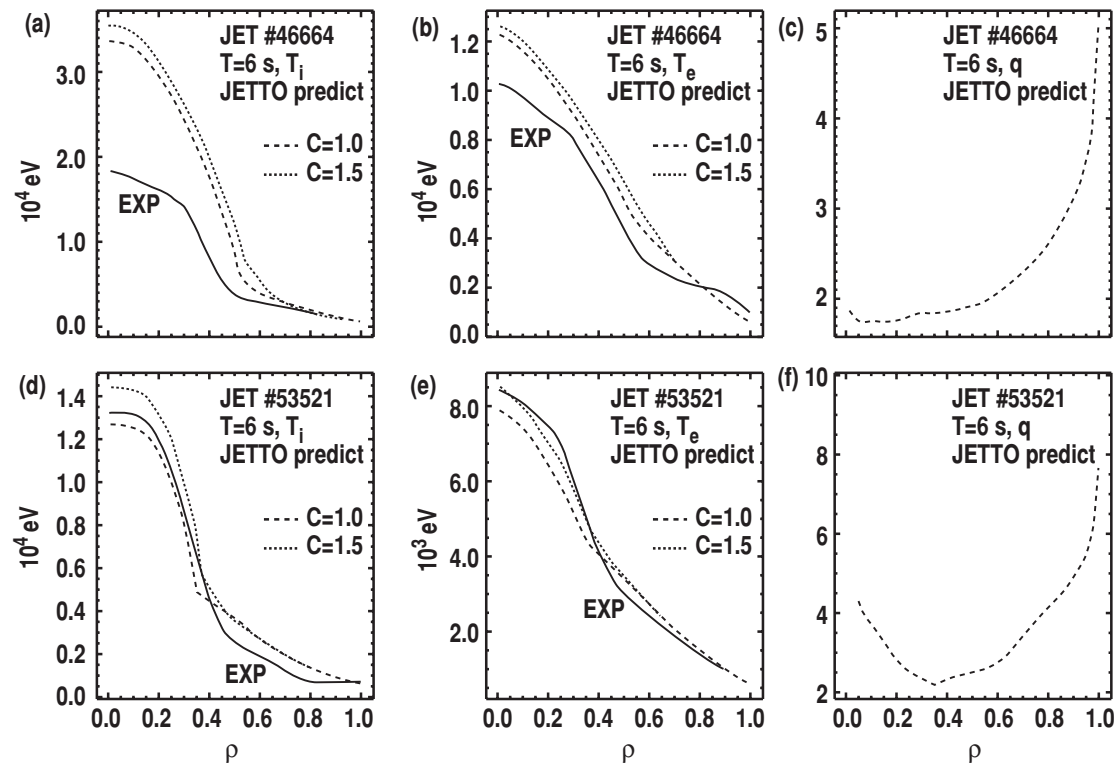

Figure 4. Comparisons between predicted results from the JETTO transport model and experimental results for the $T_{\mathrm{i}}$ and $T_{\mathrm{e}}$ profiles in ITB discharges in JET. The $q$-profiles are the predicted values from JETTO. $(a)-(c)$ correspond to weak positive central magnetic shear whilst $(d)-(f)$ correspond to strong negative magnetic shear.
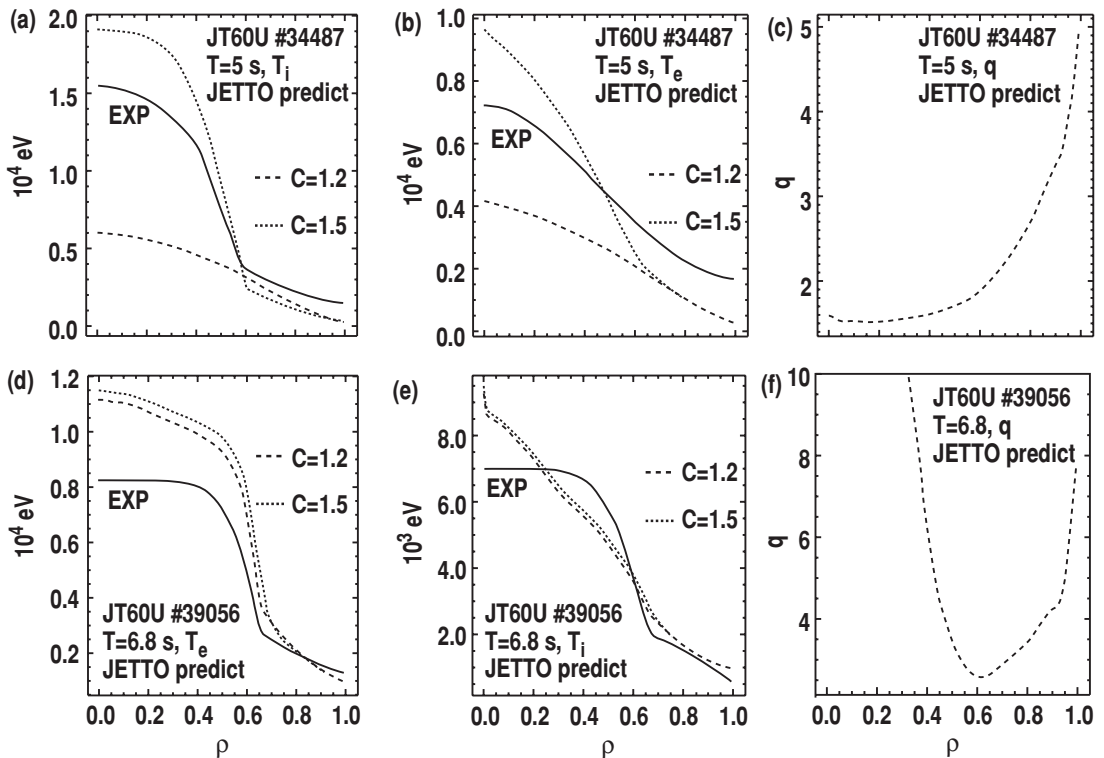

Figure 5. Comparisons between predicted results from the JETTO transport model and experimental results for the $T_{\mathrm{i}}$ and $T_{\mathrm{e}}$ profiles in ITB discharges in JT-60U. The $q$-profiles are the predicted values from JETTO. (a)-(c) correspond to weak positive central magnetic shear whilst $(d)-(f)$ correspond to strong negative magnetic shear.

experimental profiles until the multiplier $C$ exceeds a value of 1.4 (figure $5(a)$ ), indicating that the level of experimental toroidal rotation (i.e. $E \times B$ shearing rate) in this discharge is too low to trigger the ITB as predicted by the model for the given (i.e. positive) magnetic shear in the plasma. However, if the central magnetic shear is sufficiently negative (shot 39056), the model produces an ion ITB (although not the absolute central ion temperature) without any increased influence from the toroidal rotation and the $E \times B$ shearing rate, but again the model fails to produce the electron ITB.

From this multi-machine comparison, the JETTO model shows fair agreement in predicting the ion temperature profile (i.e. the ion ITB) for discharges with strong negative magnetic shear for all the devices. The predictions for the electron temperature profiles are not so good, only showing agreement with JET $T_{\mathrm{e}}$ profiles in discharges with strong magnetic shear, since the model does not include certain effects such as stabilization by the Shrafranov shift. It appears that this model is able to trigger and reproduce the ion ITB as a result of strong negative magnetic shear without a strong contribution from the $E \times B$ shearing rate. However, the model grossly overestimates both the $T_{\mathrm{i}}$ and $T_{\mathrm{e}}$ temperature profiles with low magnetic shear for DIII-D and JET, indicating the preference of the model towards exhibiting greatly reduced transport in the presence of low magnetic shear (either positive and negative) and moderate levels of plasma rotation (and hence $E \times B$ 

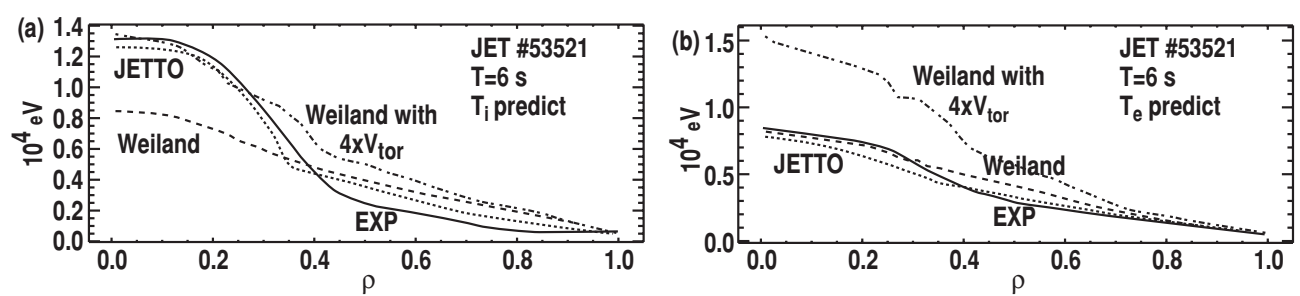

Figure 6. Comparisons between the predicted values of the $T_{\mathrm{i}}$ and $T_{\mathrm{e}}$ profiles from the JETTO and Weiland models compared to the experimental results for JET discharge 53521. (a) For the $T_{\mathrm{i}}$ profile and $(b)$ for the $T_{\mathrm{e}}$ profile.

shearing rate). Where there is both low or positive magnetic shear and low plasma rotation, as in the JT-60U monotonic $q$-profile shot, then the model underestimates the temperature profiles and requires significant increase in the $E \times B$ shearing rate to produce the ITB. This disparate behaviour for discharges from the above devices indicates one of the deficiencies of the transport model in the use of the Heaviside step function to parametrize the transport coefficients. The models need to incorporate a more gradual onset of the variable contribution of central magnetic and the $E \times B$ shearing rate in order to better model the discharges from many devices, rather than an abrupt change which results from the use of the Heaviside step function.

The Weiland model was less successful in reproducing the ITBs when run in predictive simulations. The result of a simulation with the strong negative shear discharge from JET (shot 53521) is shown in figure 6. The simulation results do not agree with the experimental data for both the $T_{\mathrm{i}}$ and $T_{\mathrm{e}}$ profiles. Improved agreement is obtained for the $T_{\mathrm{i}}$ profile if the toroidal rotation (and, hence, the $E \times B$ shearing rate) is increased by a factor of 4 or, similarly, if the ITG growth rates are reduced by a factor of 4 . However, this increase then overestimates the $T_{\mathrm{e}}$ profile. Since the actual experimental toroidal rotation profiles were used in the analysis, the lack of agreement is primarily due to the poor treatment of the $E \times B$ flow shear suppression in the model.

The results of the predictive simulations using the JETTO and Weiland models are indicative of the general status of the modelling of ITBs by a number of models. That is, whereas there may be some agreement between simulations and experimental results for some conditions, the models fail when applied over a wide range of conditions, indicating limited predictive capabilities. In particular, the agreement between experimental and modelled results observed for certain devices disappears when applied over a range of machines. The present analysis demonstrates why comparative tests and validations of models using data from many devices are required and show the need for experimentalists and modellers to work closely together to improve on the models.

\section{Gyrokinetic stability analysis of ITB plasmas}

The gyrokinetic stability analysis of ITB discharges was also carried out for the six discharges from DIII-D, JET, and JT-60U. The maximum linear ITG/TEM mode growth rates were obtained using the GKS gyrokinetic code [15] including non-circular geometry and electromagnetic effects. Figure 7 shows $\gamma_{\max }$ versus normalized radius for each of the six cases along with the computed $E \times B$ shear rate, $\omega_{E \times B}$. Here,
$\omega_{E \times B}$ was computed taking the toroidal rotation and pressure gradient from experimental data and computing the poloidal rotation from neoclassical theory to construct the radial electric field $E_{r}$ in the radial force balance equation. While $E \times B$ shear is effective in suppressing the long wavelength (low- $k$ ) ITG growth rates, alpha stabilization (Shafranov shift) is effective in suppressing both the low- $k$ ITG and high- $k$ ETG modes by reducing the geodesic curvature drive for weak or reversed magnetic shear conditions. Shafranov shift can also lead to suppression of TEM modes via drift reversal but TEM modes were not found to be important in the cases examined here with the exception of JET discharge 53521 at $\rho=0.6$. In general, we find that the magnitude of the ITG mode growth rates near the half-radius is significantly lower for the NCS discharges compared to the monotonic $q$-profile discharges. The level of heating and momentum input then needed to produce an ITB is therefore reduced for reversed magnetic shear conditions. The gyrokinetic stability analysis shows that the $E \times B$ shear rate is comparable to the maximum linear growth rate for drift-wave instabilities at the location of the ITB. This is consistent with $E \times B$ flow shear suppression of turbulence being a vital contribution for the formation of transport barrier in all three devices. A comparison between the Hahm-Burrell $\left(R B_{\mathrm{p}} / B\right) \mathrm{d} / \mathrm{d} r\left[E_{r} /\left(R B_{\mathrm{p}}\right)\right][6]$ and Waltz flux-surface averaged $\left[(r / q) / \mathrm{d} r\left(E_{r} /\left(R B_{\mathrm{p}}\right)\right] E \times B\right.$ shear rates [10] for the DIII-D and JET discharges demonstrates significant difference between the two formulae, particularly in the outer half of the plasma. This is due to the pre-derivative factor $(r / q)$ which can be significantly smaller in elongated plasmas than the corresponding factor $\left(R B_{\mathrm{p}} / B\right)$ commonly used in the Hahm-Burrell formula as discussed in [16]. For circular, large aspect ratio (typically for low beta) plasmas the two quantities are the same. For real geometry (e.g. elongation $=2$, aspect ratio $=3$ ), the Hahm-Burrell rate can be 2-3 times greater than the Waltz rate. In toroidal geometry the $E \times B$ stabilization process involves flux surface averaging with ballooning modes rotating from good to bad curvature and back. Hahm-Burrell defines a rate on the outboard side (starting with slab-like equations) which is higher than the Waltz rate mainly because Hahm-Burrell is not a flux surface quantity whereas Waltz is a flux surface function (i.e. constant on a flux surface). In general, the Waltz $E \times B$ shearing rate is more appropriate across the whole plasma, although the Hahm-Burrell shearing rate is adequate inside $\rho=0.5$, but is relatively high for $\rho>0.5$.

Further extension of this work is to increase the number of different stability codes used for the comparison of the calculated linear growth rates in the different devices. This work is presently underway using the FULL [17] and 

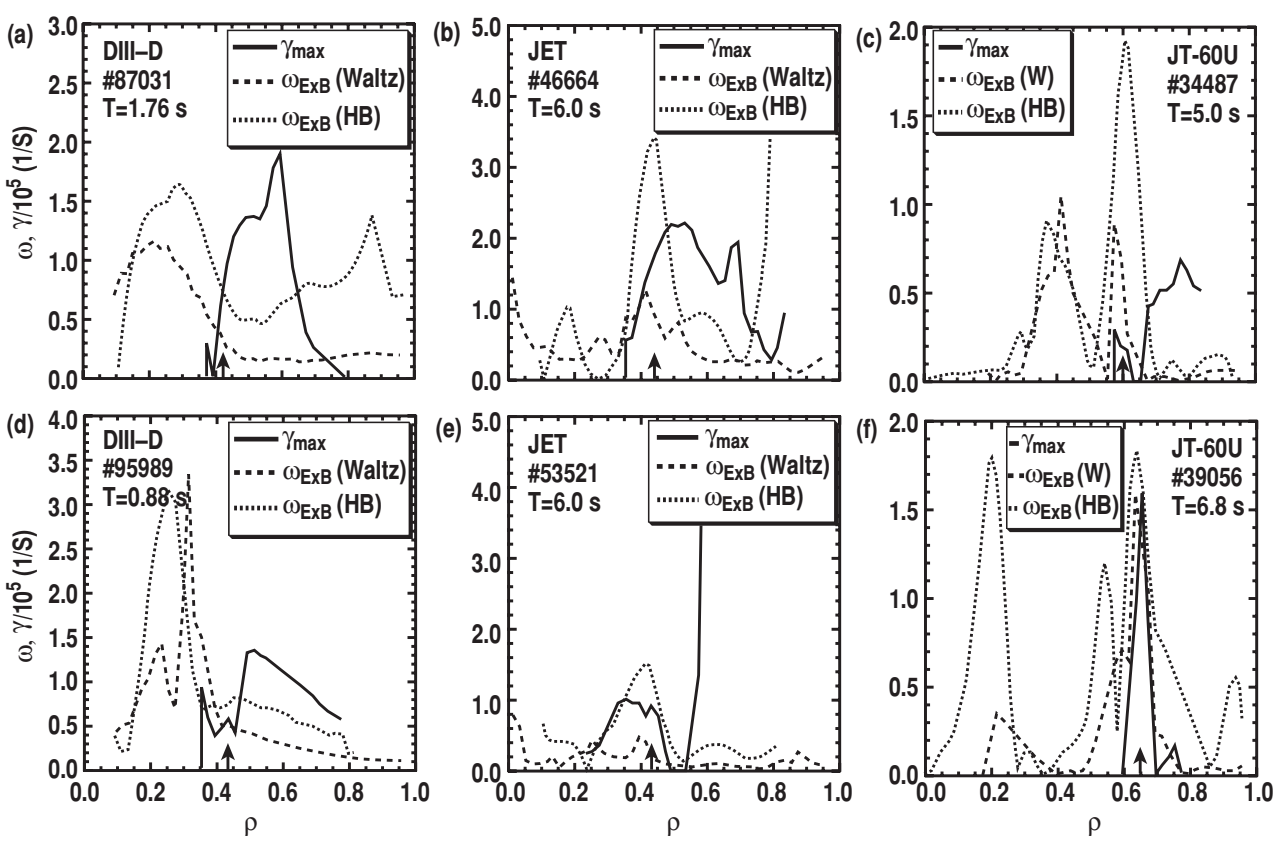

Figure 7. Comparisons between the radial profiles of the $E \times B$ shearing rate and the ITG/TEM growth rates for weak negative/positive magnetic shear and strong negative magnetic shear in DIII-D, JET, and JT-60U. The arrows indicate the location of the $T_{\mathrm{i}}$ ITB.

KINEZERO [18] gyrokinetic stability codes. All these comparisons can reveal trends that need to be determined in order to make extrapolations to future devices. It should be noted that quantitative differences in the calculation of the growth rates will occur for the different codes as a result of: (a) electrostatic versus electromagnetic treatment of the modes; (b) differences in the input of geometric effects and different MHD equilibrium codes; (c) inclusion (or non-inclusion) of suppression mechanisms such as alpha stabilization. However, one of the purposes of these comparisons is to reveal the reasons for the differences in order to provide more consistent evaluations of the growth rates across many devices.

\section{Summary}

An increased understanding of the physics of ITBs is being obtained through analysis and comparison of experimental data from a number of tokamaks. The international ITBDB provides the basis for performing these multimachine comparisons aimed at determining the requirements for the formation and sustainment of ITBs. Analysis using the database indicates that the power required to form ITBs decreases with increased negative central magnetic shear of the target plasma. Also, results from the database indicate that the $E \times B$ shear rate is close to the linear growth rate of the ITG modes at the time of barrier formation across several fusion devices thus stressing the importance of producing sufficient flow shear for barrier formation for the given level of growth rates. The JETTO transport model is able to reproduce $T_{\mathrm{i}}$ and $T_{\mathrm{e}}$ ITBs in strong negative magnetic shear plasmas in JET. However, tests of the JETTO and Weiland transport models using the two-dimensional profile data from the database indicates that there is only limited agreement between the model predictions and experimental results when considered over a range of plasma conditions in DIII-D, JET, and JT-60U. These multi-machine tests of the transport models indicate that improvements in the models are required in order to enhance their predictive capability. These improvements can result from more accurate and reliable treatment of the transport suppression mechanisms such as $E \times B$ flow shear, negative magnetic shear and alpha stabilization in the models. However, clearly more interaction is required between modellers and experimentalists to incorporate these improvements and perform more tests of the models; this will be the subject of future work. Gyrokinetic stability analysis of the ITB discharges indicates that the ITG/TEM growth rates decrease with increased negative magnetic shear, being consistent with the reduced input power requirements for ITB formation with increased negative magnetic shear, and that the $E \times B$ shear rate is comparable to the linear growth rate at the location of the ITB.

\section{Acknowledgments}

Work supported by US Department of Energy under Contracts DE-AC03-99ER54463, DE-AC02-76CH03073, and Grants DE-FG03-01ER54615, DE-FG02-92ER54141, DE-FC02-99ER54512.

\section{References}

[1] Fukuda T. et al 2001 Proc. 28th EPS Conf. (Maderia, Spain)

[2] Sips A.C.C. et al 2002 Plasma Phys. Control. Fusion 44 A391

[3] Baranov Y. 2001 Bull. Am. Phys. Soc. 46117

[4] Hoang G.T. et al 2002 Proc. 29th EPS Conf. (Montreux, Switzerland)

[5] Fukuda T. et al 2002 Proc. 29th EPS Conf. (Montreux Switzerland)

[6] Hahm T.S. and Burrell K.H. 1995 Phys. Plasmas 21648

[7] Newman D.E. et al 1998 Phys. Plasmas 5938

[8] Burrell K.H. 1997 Phys. Plasmas 41499 
[9] Weiland J. 2000 Collective Modes in Inhomogeneous Plasmas (Bristol: Institute of Physics Publishing)

[10] Waltz R.E. et al 1997 Phys. Plasmas 42482

[11] Cennachi G. and Tami A. 1988 JET-1R(88), 03

[12] Waltz R.E. 2002 General atomics, Private communication

[13] Erba M. et al 1997 Plasma Phys. Control. Fusion 39261
[14] Houlberg W.A. et al 1997 Phys. Plasmas 43230

[15] Kotschenreuther M. et al 1995 Comput. Phys. Commun. 88 128

[16] Waltz R.E. and Miller R.L. 1999 Phys. Plasmas 64265

[17] Rewoldt G. et al 1997 Phys. Plasmas 43293

[18] Bourdelle C. et al 2002 Nucl. Fusion 42892 